\title{
Elektromagnetische Felder bei Patienten mit chronischen Rückenschmerzen (low-back pain): Eine doppelblinde randomisierte Duo-Center-Studie
}

\author{
Günther Bernatzky1, Werner Kullich2, Ferdinand Aglas², Michael Ausserwinkler3, Rudolf Likar4, \\ Wolfgang Pipam4, Helmut Schwann², Wolf A. Kafka ${ }^{5}$
}

${ }^{1}$ Fachbereich für Organismische Biologie, Naturwissenschaftliche Fakultät, Universität Salzburg, Österreich; 2Ludwig-Boltzmann Cluster für Rheumatologie, Balneologie und Rehabilitation; Institut für Rehabilitation interner Erkrankungen, Saalfelden, Österreich; ${ }^{3}$ Ludwig Boltzmann Cluster für Rheumatologie, Balneologie und Rehabilitation, Aussenstelle Althofen, Österreich; 4Interdisziplinäre Schmerzambulanz, Klagenfurt, Österreich; 5EMPHYSPACE International Research Association, Kottgeisering, Deutschland.

$D^{\prime}$ ie Effekte der therapeutischen Anwendung von Elektromagnetfeldern sind Gegenstand intensiver Forschungsarbeiten. Jedoch ist es bis heute noch nicht gelungen, alle biologischen Mechanismen dieser Therapieform zu erkennen. Daher ist es umso wichtiger, kritische Untersuchungen über spezifische Aspekte von Gewebsfunktionen mit präzisen Methoden bezüglich der Auswirkungen schwacher, nicht ionisierender elektromagnetischer Felder, die extern appliziert werden, zu untersuchen.

Angesichts der alarmierenden $\mathrm{Zu}-$ nahme chronischer Schmerzen des Stütz- und Bewegungsapparates und der entsprechenden Suche nach neuen kausalorientierten, präventiven, insbesondere kostenreduzierenden Massnahmen kommen zunehmend auch elektromagnetische Interventionen zum Einsatz. Deren therapeutische Erfolge gelten - bis auf einige Ausnahmen $[1,6,11,13-17,20,21,22,24,28,29,31]$ jedoch aufgrund nicht evidenzbasierender Untersuchungen, vor allem aber wegen der vielen, auf ungeprüfte Hypothesen aufgesetzten Heilversprechen, insbesondere zur Mikrozirkulation, als ungesichert $[2,10,18,25]$.

$\mathrm{Zu}$ den Ausnahmen zählen interessanterweise solche Untersuchungen, die sich auf speziell konzipierte, spektral breit zusammengesetzte Stimulationssignale stützen. Ausgerichtet auf die Unterstützung natürlich vorgegebener Regulationsmechanismen zur organismischen Selbsterhaltung und
Hintergrund: Die alarmierende Zunahme chronischer Schmerzen des Stütz- und Bewegungsapparates gibt Anlass zur Suche nach neuen kausalorientierten, präventiven, insbesondere kostenreduzierenden Massnahmen. Hierzu gehören neben der reinen Schmerzbehandlung auch präventive Massnahmen zur Beeinflussung der die Grösse der Infarktareale beeinflussenden Adhäsionsmoleküle (PECAM-1, Platelet-endothelial Cell Adhesion Molecule, und P-Selektin). Zielsetzung: Ausgerichtet auf die Behandlung von Patienten mit chronischem Kreuzschmerz sollte in diesem Zusammenhang untersucht werden, ob und inwieweit eine zu etablierten, standardisierten Rehabilitationsverfahren additive nicht-invasive Intervention mit speziell konzipierten elektromagnetischen Stimulationssignalen grosser spektraler Bandbreite hier hinsichtlich der folgenden Zielparameter zu Verbesserungen führen kann: schmerzende Störungen des Stütz- und Bewegungsapparates, Depression, Angst, Schlafqualität, Infarktareale beeinflussende Adhäsionsmoleküle (PECAM-1 und P-Selektin). Methoden: An der randomisierten, doppelblind placebokontrollierten Duo-Center-Studie (GCP-Standard, Untersuchungszeitraum 3 Wochen) beteiligten sich 44 Patienten mit chronischen Rückenschmerzen (lowback pain). Die elektromagnetische Intervention erfolgte additiv zu einem standardisierten Rehabilitationskonzept über flexible Ganzkörper-Elektromagnetfeldmatten. Zur Bewertung wurden validierte Beweglichkeitsmessungen, Fragebögen und enzymimmunologische Analysen herangezogen. Ergebnisse: In beiden Gruppen zeigten sich signifikante Verbesserungen von Ruhe-, Bewegungs-, Druckschmerz, Angst und Depression. Die elektromagnetische Behandlung führte darüberhinaus zu einer weiteren signifikanten Verbesserung der Beweglichkeit (Finger-Fussbodenabstand) und der Schlafqualität sowie einer signifikanten Verminderung des Adhäsionsmoleküls PECAM-1 in beiden Gruppen, nicht aber des P-Selektins. Schlussfolgerungen: Die erfolgreichen Therapiemassnahmen im Verlauf des 3-wöchigen Rehabilitationsaufenthaltes dürften die Wirkungen der elektromagnetischen Intervention teilweise maskieren. Trotz der gefundenen signifikanten Effekte der elektromagnetischen Behandlung sind diese für eine praxisrelevante Anwendung jedoch noch nicht ausreichend genug. Neben einer verlängerten Anwendungsdauer von mehr als 3 Wochen könnte sich auch auf Grund derzeitiger Forschungs- und Weiterentwicklung der gewählten Signalform eine Optimierung der Therapie einstellen. Insofern erscheint eine derartige Behandlungsform als adjuvante Massnahme zur Verbesserung von Schmerz, Schlaf und schmerzbedingter Angst und Depression bei chronischem Kreuzschmerz geeignet.

Schlüsselwörter: Low-back pain, Elektromagnetische Intervention, Stimulationssignal, spektrale Bandbreite, Rehabilitationsprogramm

\section{Electromagnetic Fields in Patients with Chronic Low-Back Pain: A Double-Blind Randomized Placebo-Controlled Two-Centre Study}

Background: The alarming increase in the number of persons afflicted with chronic back pain and impaired musculoskeletal systems asks for new, specific, preventive and especially cost-effective therapeutic methods that should focus not only on the 
sole treatment of pain but also on factors influencing the infarction area such as platelet endothelial cell adhesion molecule-1 (PECAM-1) and P-selectin. Aim of the Study: By determining the parameters of painful disturbances of the locomotion system, depression, anxiety, sleep quality, and the amount of adhesion molecules influencing the infarction area (PECAM-1 and P-selectin), the authors investigated whether patients suffering from chronic low-back pain could benefit from non-invasive, specially designed pulsed electromagnetic field applications along with a well-established standardized rehabilitation programme. Methods: A randomized double-blind, two-centre study was conducted in 44 patients with chronic low-back pain. The study period was 3 weeks. In addition to a standardized rehabilitation program, the patients underwent electromagnetic stimulation with specially designed electromagnetic fields applied via a flexible mattress. The evaluation was based on validated forms of mobility measurements, questionnaires and enzymatic and immunological analyses. Results: Significant improvements in resting, movement and pressure pain as well as in anxiety and depression were observed in both groups. Significantly exceeding the effects of the standardized rehabilitation programme, electromagnetic stimulation led to additional significant improvements in mobility (finger-floor distance) and quality of sleep, and decreased the serum levels of PECAM-1 but not of P-selectin. Conclusions: The successful therapeutic measures achieved by the 3-week rehabilitation program partly mask the effects of the electromagnetic stimulation. Current research and development aimed at optimizing the shape of the electromagnetic signals, and thus their therapeutic efficiency, could obviously lead to further improvements also in view of health care costs in the near future. This non-invasive electromagnetic intervention can thus be recommended as an adjuvant treatment administered along with a standard rehabilitation programme to patients with low-back pain.

Key words: Low-back pain, electromagnetic intervention, stimulation signal, spectral bandwidth, rehabilitation programme

-heilung soll dadurch ein möglichst breites Band der an diesen Prozessen beteiligten molekularen Interaktionen aktiv unterstützt werden [12,14]. Bezüglich der analgetischen Effekte einer Magnetfeldtherapie wurde bereits vor mehr als 20 Jahren in einer doppelblinden placebokontrollierten Studie bei Patienten mit muskuloskelettalen Beschwerden eine 3,5-mal so häufige Schmerzreduktion in der Verumgruppe im Vergleich zur Kontrollgruppe erzielt [30].

Ziel der vorliegenden Studie war es, im Rahmen eines GCP-konformen Studiendesigns $\mathrm{zu}$ prüfen und quantitativ $\mathrm{zu}$ bewerten, ob und inwieweit sich dieses Konzept mit den oben beschriebenen elektromagnetischen Stimulationssignalen auch in der Behandlung chronisch schmerzender Störungen des Stütz- und Bewegungsapparates (insbesondere bei Patienten mit chronischem Kreuzschmerz, sog. low-back pain) als praxisrelevant bewährt. Im Detail sollte im Rahmen einer randomisierten placebokontrollierten, doppelt verblindeten Studie mit einer duozentrischen Verlaufsbeobachtung in Form einer quantitativen Mehrpunktanalyse hierzu die Effizienz einer ent- sprechend konzipierten elektromagnetischen Stimulationsform quantifiziert und statistisch gesichert werden. Dabei galt es, die Differenz zwischen Placebo- bzw. Verumwerten für Parameter des Schmerzempfindens, der Depression, Angst, Schlafqualität sowie relevanter biochemischer Labordaten (zur Indikation kardiovaskulär bedeutsamer Adhäsionsmoleküle) vor und nach einem dreiwöchigen Rehabilitationsaufenthalt festzuhalten. Schmerz und Schmerzempfindung ist eine biopsycho-soziale Einheit. Die Beweglichkeit wird durch Schmerz eingeschränkt. Die Intensität der Schmerzempfindung hängt massgeblich von den begleitenden Grössen, wie Depression, Angst und Schlafqualität, ab. Da Schmerz ein hoher Stressfaktor mit erhöhtem Auftreten von Katecholaminen ist, galt es zu prüfen, ob zusätzlich unter der Magnetfeldtherapie Einflüsse auf bestimmte Adhäsionsmoleküle (PECAM-1 und P-Selektin) nachweisbar sind. Diese Moleküle haben insbesondere bei der Zellinteraktion von thrombotischen, aber auch inflammatorischen vaskulären Prozessen eine wichtige Bedeutung. Gerade P-Selektin ist Mediator der Thrombozyten-Endothel-Interaktio- nen. Ein im Rahmen einer Magnetfeldtherapie nachgewiesener inhibitorischer Einfluss auf P-Selektin und das Thrombozyten-Endothel-Adhäsionsmolekül PECAM könnte die bisher nicht vollständig bestätigten Hinweise auf unterstützende Effekte in der Mikrozirkulation erhärten. Ohne direkten Bezug zu den Wirbelsäulenaffektionen, jedoch mit dem Hintergrund, dass der behandelnde Arzt immer häufiger mit der Komorbidität von Herzkreislauferkrankungen konfrontiert ist und diese auch als mögliches Einsatzgebiet der Anwendung elektromagnetischer Felder angesehen werden, erschien es auch aus diesem Grund von Interesse, diese speziellen Adhäsionsmoleküle, die bei der Plättchen-Gefässwand-Interaktion eine grosse Rolle spielen, zu untersuchen.

Letztlich galt es nachzuweisen, dass die komplementär zum Rehabilitationsverfahren eingesetzte elektromagnetische Stimulation die Rehabilitationserfolge signifikant verbessert.

\section{Material und Methoden}

\section{Elektromagnetische Stimulation}

Innerhalb des Untersuchungszeitraums von 3 Wochen erfolgte die elektromagnetische Stimulation über speziell angesteuerte flexible Ganzkörper-Elektromagnetfeldmatten $(\mathrm{L} / \mathrm{B} / \mathrm{J}=170 / 80 / 3 \mathrm{~cm})$ gemäss Programm P4 des Systems BEMER3000 (Innomed International AG, FL-Triesen) liegend im Krankenzimmer zweimal täglich für eine Dauer von 20 Min. (vormittags $8.00 \mathrm{Uhr}$ bis 8.20 Uhr und abends 17.00 Uhr bis 17.20 Uhr). Insgesamt sind die Patienten damit 40 Min. täglich auf der Verum- bzw. Placebomatte gelegen.

Die in den Matten integrierten Flachspulen (4 Kreis- und 2 Ovalflachspulen, Durchmesser ca. 30/30x50 cm) lieferten einen stark inhomogenen und in seiner spektralen Zusammensetzung breitbandig mit einem pulsierenden von 0 bis max. 35 mikroTesla ansteigenden Verlauf der magnetischen Flussdichte gemäss $\mathrm{B}(\mathrm{t})=\mathrm{t}^{\wedge} 3^{*} \mathrm{e}^{\wedge} \sin \left(\mathrm{t}^{\wedge} 3\right)$, mit $\mathrm{t} \in$ [1:4] (Pulsdauer $30 \mathrm{~ms}$; Pulshäufigkeit 30/s) [12] (dieser Wert liegt unterhalb der örtlich gegebenen geomagnetischen Flussdichte von ca. 50 mikroTesla). 


\section{Patienten}

Die Untersuchungen wurden im Rahmen eines standardisierten, GCP-konformen Designs in 2 Zentren durchgeführt. Es handelte sich um stationär aufgenommene Patienten $(n=44, m=20$, w=24; Tab. 1) mit chronischen Kreuzschmerzen (low-back pain) aufgrund eines computertomographisch verifizierten Discusprolaps (Z. ohne Operation, Scorewert der VAS>4) in den Rehabilitationszentren a) Sonderkrankenanstalt für Erkrankungen des Stützund Bewegungsapparates und HerzKreislauferkrankungen der PVA, Saalfelden, und b) Privatklinik St. Barbara, Med. Zentrum, Bad Vigaun.

Die Patienten im Alter von 23 bis 71 $(51,9 \pm 10,1)$ Jahren erhielten im Rahmen der stationären Rehabilitation ein klar definiertes und auf chronischen Kreuzschmerz zugeschnittenes Therapieprogramm (Tab. 1):

- Wirbelsäulengymnastik-Gruppentherapie: pro Trainingseinheit 30 Min.; 2x pro Woche,

- Unterwassergymnastik-Gruppentherapie: pro Trainingseinheit

10 Übungen zu je 2 Min.; 2x pro Woche,

- Therapeutisches Schwimmen: 20 Min. täglich,

- Parafango/Therapie: 20 Min.; pro Einheit 1-2x pro Woche,

- Rückenmassagen: 20 Min.; 2x pro Woche,

- Rückenschule: 60 Min.; 2x pro Woche.

Zur Reduktion potentieller Störeinflüsse auf die applizierten elektromagnetischen Felder waren elektrotherapeutische Anwendungen an der Wirbelsäule und Stangerbäder bei den Probanden ausgeschlossen.

$\mathrm{Zu}$ Beginn der Studie erfolgte eine mündliche und schriftliche Patienteninformation sowie Einholung der Einverständniserklärung der Patienten. Die Studie wurde entsprechend der Deklaration des Weltärztebundes von Helsinki «Ethische Grundsätze für die medizinische Forschung am Menschen» (Tokio, 2004) durchgeführt. Je 10 männliche und 12 weibliche Patienten wurden gemäss einer Randomisierungstabelle je einer Verum- (= Matte mit Feldwirkung + Therapieprogramm)

Tab. 1. Demografische Daten der Patienten

\begin{tabular}{|c|c|c|c|c|c|}
\hline & & \multicolumn{2}{|c|}{$\begin{array}{l}\text { Verumgruppe }(n=22) \\
\text { Elektro-Magnetfeld-Aktiv }\end{array}$} & \multicolumn{2}{|c|}{$\begin{array}{l}\text { Placebogruppe }(n=22) \\
\text { Elektro-Magnetfeld-Inaktiv }\end{array}$} \\
\hline & & \multicolumn{2}{|c|}{10 Männer, 12 Frauen } & \multicolumn{2}{|c|}{10 Männer, 12 Frauen } \\
\hline & & - & s & $\begin{array}{l}- \\
\mathbf{x}\end{array}$ & s \\
\hline Alter & Jahre & 50.20 & 11.80 & 53.60 & 8.00 \\
\hline Größe & $\mathrm{m}$ & 1.69 & 0.09 & 1.69 & 0.07 \\
\hline Gewicht & $\mathrm{kg}$ & 77.80 & 13.80 & 74.70 & 14.10 \\
\hline Body mass-Index & BMI & 27.00 & 3.80 & 26.10 & 3.70 \\
\hline Beschwerdedauer & Monate & 126 & 91 & 101 & 90 \\
\hline
\end{tabular}

Tab. 2. Gesamtprocedere der Studie

1. Einschluss: Aufnahmegespräch

Tag 1: Case report Form, Ärztl. Aufnahmeuntersuchung, Aufnahmelabor

2. HADS-D Fragebogen, KKG Fragebogen, Roland-Morris-Fragebogen, PSQIFragebogen, SF-36-Fragebogen

3. nach 1 Woche: 1. Ärztl. Zwischenuntersuchung, SF36

4. nach 2 Wochen: 2. Ärztl. Zwischenuntersuchung, SF36

nach 3 Wochen: Ärztl. Abschlussuntersuchung: HADS-D Fragebogen, KKG-

5. Fragebogen, Roland-Morris-Fragebogen, PSQI-Fragebogen, SF-36-Fragebogen. Abschluss Case report Form

bzw. einer Placebo- (= Matte ohne Feldwirkung + Therapieprogramm) Gruppe zugeordnet. Dabei wurden verblendete Elektromagnetfeldmatten verwendet. Weder Patienten noch Ärzte oder sonstiges Personal konnten feststellen, welche Patienten auf der Verummatte oder auf der Placebomatte lagen. Bei sonst gleichen Versuchsbedingungen war bei der Placebogruppe - für den Patienten nicht erkennbar - das Feld nicht aktiviert. Während des Liegens hatten die Patienten beider Gruppen die Möglichkeit, entspannt zu lesen.

\section{Zielparameter / Versuchsprotokoll}

Die Bestimmung der Zielparameter erfolgte gemäss eines definierten Versuchsprotokolls (Tab. 2) vor und nach der 3-wöchigen Anwendung der aktiven bzw. inaktiven Applikationen.

Schmerz wurde in Ruhe und in Bewegung mittels 10-teiliger Visueller Analogskala, Druckschmerz mittels 4teiliger verbaler Rating-Skala bestimmt. Der Finger-Fussbodenabstand sowie der Laterale Finger-Fussbodenabstand wurden bei den Visiten gemessen. Weiters wurde der Roland \& Morris-Disability Questionnaire (RMDQ) [23] eingesetzt, der mittels 24 Teilfragen die 
funktionelle Behinderung durch Rückenschmerzen beurteilt. Die Analyse von Veränderungen von Schlafstörungen wurde an Hand von 24 Teilfragen des Pittsburgh Sleep Quality-Index (PSQI) [5] vorgenommen. Die Hospital Anxiety and Depression-Scale (HADSD, deutsche Version) [8,9,34] diente zur Identifizierung und Quantifizierung von Angst und Depression. Kontrollüberzeugungen zu Krankheit und Gesundheit (KKG-Bogen) wurden mit dem Fragebogen von LOHAUs und Sснмітт [19], gesundheitsbezogene Angaben zur „körperlichen“ und „psychischen Gesundheit“ mittels des Schmerzfragebogens (SF-36) [4] erfasst.

\section{Laboranalysen von PECAM-1 und P-Selektin}

Die Ermittlung der Adhäsionsmoleküle erfolgte im Rahmen von Routineblutabnahmen zu Beginn und am Ende des Rehabilitationsaufenthaltes aus Serum-/Plasma entnommenen Blutproben. Mittels enzymimmunologischer Methoden wurden die Adhäsionsmoleküle P-Selektin [3] (Granule Membran Protein-140; Human sP-selectin ELISA, Fa. Bender MedSystems GmbH, Österreich) und PECAM-1 (Platelet Endothelial Cell Adhesion Molecule-1; Human sPECAM-1 ELISA, Fa. Bender MedSystems $\mathrm{GmbH}$, Österreich) aus den bei $-50^{\circ}$ $\mathrm{C}$ gelagerten Serumproben bestimmt.

\section{Statistische Auswertung}

Die EDV-mässige Datenerfassung und die statistische Auswertung erfolgte mit Hilfe des Tabellenkalkulationsprogrammes MS Excel 2003 (Microsoft, USA) und der Statistik-Programmpakete SYSTAT 9.0 Statistics for Windows (SPSS Incorp., USA) und SPSS Vs. 12.0.1 for Windows (SPSS Incorp., USA). Dabei wurden folgende Testverfahren angewandt: Descriptive Statistik, paarweiser Wilcoxon U-Test, Student-t-Test sowie Regressionsanalyse zur Berechnung kausaler Zusammenhänge.

\section{Ergebnisse}

Sowohl in der Verumgruppe als auch in der Placebogruppe zeigten sich gegenüber den Ausgangswerten (Kon- trolle) nach Behandlungsablauf signifikante Besserungen der Schmerzparameter Ruheschmerz, Bewegungsschmerz und Druckschmerz (Tab. 3): Gemessen an dem signifikant verringerten mittleren Finger-Fussbodenabstand von $11,2 \mathrm{~cm}$ auf $7,7 \mathrm{~cm}(\mathrm{p}<0,01)$ führte die Verumbehandlung gegenüber der Placebogruppe in der Verumgruppe zu einem signifikant über den Placeboeffekt (n.s.) hinausgehenden Behandlungsvorteil. Dieser klare Vorteil ergab sich insbesondere ausschliesslich auch für die durch die Verumbehandlung über den PSQI-Fragebogen ermittelte induzierte hochsignifikant verbesserte Schlafqualität $(\mathrm{p}<0.001)$. Der Roland \& Morris-Score für low-back pain [23] verbesserte sich sowohl unter Verum $(\mathrm{p}<0,03)$ als auch unter Placebo $(\mathrm{p}<0.02)$ signifikant. Auch Angst und Depression, gemessen mittels HADS-D-Skala, verminderten sich in beiden Behandlungsgruppen signifikant $(\mathrm{p}<0.01)$.

Mittels KKG wurde die Kontrollüberzeugung zu Krankheit und Gesundheit quantifiziert [19]: In insgesamt 21 Fragen (Items) wurde dabei die Kontrollüberzeugung zu den drei Dimensionen Internalität, Soziale Externalität und Fatalistische Externalität erhoben, wobei bei jeder Dimension 7 Items mit je einer Beantwortungsmöglichkeit von 1 bis 6 gegeben war. Es waren statistisch relevante Verbesserungen weder in der Verumnoch in der Placebogruppe vorhanden.

Hinsichtlich des Gesundheitszustandes, erhoben mit dem SF-36 Fragebogen [4], zeigten sich keine signifikanten Unterschiede zwischen Verum- und Placebogruppe. In beiden Gruppen, also bei Rehabilitationsprogramm sowohl mit aktiver als auch ohne aktive Feldbehandlung, waren bei allen Subgruppen des Fragebogens (Körperliche Funktionsfähigkeit, Körperliche Rollenfunktion, Schmerz, Vitalität, Soziale Funktionsfähigkeit, Psychisches Wohlbefinden, Gesundheitsveränderung) von Beginn bis Ende der Therapie signifikante Unterschiede im Sinne von Verbesserungen feststellbar. Ausgenommen davon ist die Allgemeine Gesundheitswahrnehmung, die in keiner der beiden Gruppen zu statistisch relevanten Unterschieden führte. Einzig die Emotionale Rollenfunktion war in der Placebogruppe signifikant unterschiedlich $(\mathrm{p}<0.03)$.

Auch die aus den Subgruppen berechneten Körperlichen und Psychischen Summenskalen des SF-36 waren sowohl in der Verumgruppe als auch in der Placebogruppe signifikant verbessert (Tab. 4).

Die statistische Analyse ergab bei den Messungen der Adhäsionsmoleküle P-Selektin und PECAM einen statistischen Unterschied zwischen der Verum- und der Placebogruppe an beiden Messzeitpunkten. Aufgrund individueller relativ hoher Streuungen erwiesen sich die Verminderungen des Adhäsionsmoleküls P-Selektin nach 3 Wochen als nicht signifikant (Abb. 1). Hingegen waren die PECAM-Werte nach 3 Wochen in beiden Gruppen hoch signifikant verringert. Eine Korrelationsanalyse der Veränderungen (Differenzen) während der Therapiephase zeigt vor allem in der Verumgruppe interessante Zusammenhänge: So korrelierten die Verbesserungen des Ruheschmerzes mit der Reduktion des Adhäsionsmoleküls PECAM und der PSQI-Schlafqualitätsscore mit der Besserung von Bewegungsschmerz und Depression (Tab. 5).

\section{Diskussion}

Die Mikrozensus-Erhebung 1999 als wichtigste österreichische Datenquelle für die gesundheitliche Risikobelastung der Bevölkerung zeigt, dass mehr als die Hälfte der Bevölkerung eine gesundheitliche Beschwerde angibt und die häufigsten Beschwerden Rückenbzw. Kreuzschmerzen sind. Etwa ein Fünftel der Männer und Frauen (je $20,7 \%$ ) sind davon betroffen [27]. In der vorliegenden Studie wurde gezeigt, dass in beiden Gruppen sowohl der Allgemeine Gesundheitszustand (inklusive der Schlafqualität) als auch der chronische Kreuzschmerz erfolgreich behandelt wurden. Die im Rahmen der dreiwöchigen Therapie üblichen Therapieverfahren zeigen sich damit als wirkungsvoll.

Die signifikanten Besserungen der Schmerzparameter Ruheschmerz, Bewegungsschmerz und Druckschmerz 
Tab. 3. Additive Anwendung einer durch spezielle Signalformen gekennzeichneten Elektromagnetfeldtherapie während stationärer Rehabilitation

\begin{tabular}{|c|c|c|c|c|c|c|c|c|c|c|c|}
\hline & \multirow{3}{*}{ Einheit } & \multicolumn{5}{|c|}{ Elektro-Magnetfeld-aktiv (Verumgruppe; $n=22$ ) } & \multicolumn{5}{|c|}{ Elektro-Magnetfeld-inaktiv (Placebogruppe; $n=22$ ) } \\
\hline & & \multicolumn{2}{|c|}{ vor Therapie } & \multicolumn{2}{|c|}{$\begin{array}{l}\text { nach } 3 \text { Wochen } \\
\text { Therapie }\end{array}$} & \multirow{2}{*}{$\begin{array}{c}\text { Sig. } \\
p \\
\end{array}$} & \multicolumn{2}{|c|}{ vor Therapie } & \multicolumn{2}{|c|}{$\begin{array}{c}\text { nach } 3 \text { Wochen } \\
\text { Therapie }\end{array}$} & \multirow{2}{*}{$\frac{\text { Sig. }}{p}$} \\
\hline & & $\bar{x}$ & s & $\bar{x}$ & s & & $\bar{x}$ & $\mathrm{~s}$ & $\bar{x}-\bar{x}$ & $\mathrm{~s}$ & \\
\hline Ruheschmerz (VAS) & Score $0-10$ & 5.45 & 2.10 & 3.08 & 1.55 & $<0,0002$ & 4.09 & 2.32 & 2.55 & 1.58 & $<0,02$ \\
\hline $\begin{array}{l}\text { Bewegungsschmerz } \\
\text { (VAS) }\end{array}$ & Score $0-10$ & 5.52 & 2.22 & 2.90 & 1.67 & $<0,0002$ & 5.68 & 1.65 & 3.56 & 1.87 & $<0,0005$ \\
\hline Druckschmerz & Score $0-3$ & 1.59 & 1.10 & 0.77 & 0.81 & $<0,01$ & 1.09 & 1.02 & 0.55 & 0.74 & $<0,03$ \\
\hline Finger-Fußboden Abstand & $\mathrm{cm}$ & 11.20 & 12.80 & 7.70 & 10.40 & 0.01 & 10.90 & 13.90 & 9.60 & 13.00 & n.s. \\
\hline $\begin{array}{l}\text { Lateraler Finger- } \\
\text { Fußboden-Abstand }\end{array}$ & $\mathrm{cm}$ & 49.30 & 9.60 & 45.30 & 13.10 & n.s. & 51.50 & 6.30 & 46.50 & 9.00 & n.s. \\
\hline $\begin{array}{c}\text { Roland\&Morris-Score for } \\
\text { Low back pain }\end{array}$ & & 8.55 & 4.49 & 7.09 & 4.28 & $<0,03$ & 9.27 & 4.07 & 7.64 & 4.20 & $<0,02$ \\
\hline $\begin{array}{l}\text { PSQI-Schlafqualitäts- } \\
\text { Index }\end{array}$ & & 9.55 & 3.61 & 7.05 & 2.68 & $<0,001$ & 8.21 & 3.68 & 6.59 & 3.83 & n.s. \\
\hline HADS-D Angst & & 8.23 & 4.73 & 5.64 & 3.37 & $<0,01$ & 6.86 & 3.14 & 4.91 & 3.05 & $<0,01$ \\
\hline HADS-D Depression & & 5.45 & 4.01 & 4.32 & 3.23 & $<0,03$ & 4.82 & 2.17 & 3.64 & 2.59 & $<0,01$ \\
\hline
\end{tabular}

Tab. 4. SF-36 Schmerzfragebogen: Signifikante Unterschiede (t-Test) vor und nach 3-wöchiger Rehabilitation mit und ohne elektromagnetische Intervention

\begin{tabular}{|l|c|c|}
\hline \multicolumn{1}{|c|}{ SF-36 Subgruppe } & $\begin{array}{c}\text { Elektro-Magnetfeld aktiv + } \\
\text { Rehab (Verumgruppe; } n=22)\end{array}$ & $\begin{array}{c}\text { Elektro-Magnetfeld inaktiv + Rehab } \\
\text { (Placebogruppe; } n=22)\end{array}$ \\
\cline { 2 - 4 } & \multicolumn{2}{|c|}{ Signifikanz (p) } \\
\hline Körperliche Funktionsfähigkeit & $<0,03$ & $<0,01$ \\
\hline Körperliche Rollenfunktion & $<0,01$ & $<0,002$ \\
\hline Körperliche Schmerzen & $\mathrm{n} . \mathrm{s}$. & $\mathrm{n} . \mathrm{s}$. \\
\hline Allgemeine Gesundheitswahrnehmung & 0.001 & $<0,00002$ \\
\hline Vitalität & $<0,01$ & $<0,05$ \\
\hline Soziale Funktionsfähigkeit & $\mathrm{n} . \mathrm{s}$. & $<0,03$ \\
\hline Emotionale Rollenfunktion & $<0,001$ & $<0,00005$ \\
\hline Psychisches Wohlbefinden & $<0,001$ & $<0,01$ \\
\hline Gesundheitsveränderung & $<0,01$ & $<0,01$ \\
\hline Körperliche Summenskala & $<0,05$ & $<0,01$ \\
\hline Psychische Summenskala & & $<$ \\
\hline
\end{tabular}




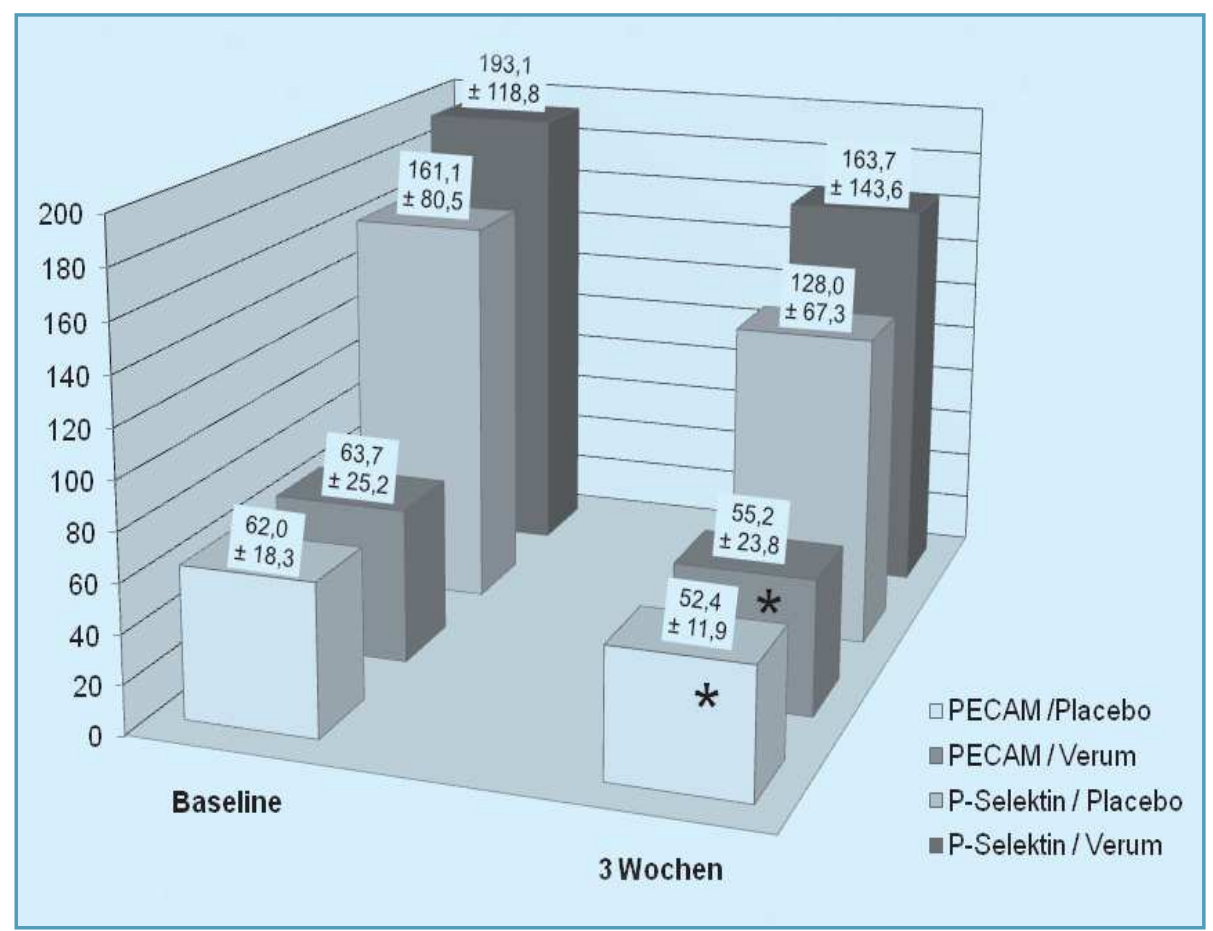

Abb. 1. Verhalten der Adhäsionsmoleküle P-Selektin und PECAM in vitro mit und ohne gepulste elektromagnetische Felder.

in beiden Gruppen dokumentiert eindrucksvoll das effiziente standardisierte Therapieprogramm in beiden an dieser Studie beteiligten Rehabilitationszentren.

Für die Beurteilung der Elektromagnetfeldeffekte ist die suffiziente stationäre Rehabilitation leider hinderlich, da eventuelle Einflüsse auf den chronischen Kreuzschmerz maskiert werden, was der statistische Vergleich der beiden Interventionsgruppen, die sich nicht signifikant unterschieden, beweist.

Der stimulierende Effekt eines pulsierenden elektromagnetischen Feldes auf den Knorpelmetabolismus wurde in mehreren In-vitro-Studien gezeigt [7]. Ein additiver Effekt der Therapie mit elektromagnetischen Feldern auf schmerzhafte Arthrosen der Zwischen- wirbelgelenke könnte klarerweise helfen, dadurch bedingte chronische Kreuzschmerzen zu bessern.

Interessant erscheint die Tatsache, dass sich bei den Patienten unter der Verumbehandlung eine signifikante Verbesserung $(p<0,001)$ der Schlafqualität, gemessen mittels des PSQI, ergab. Durch diese signifikante Verbesserung der Schlafqualität ist letztlich auch erklärbar, dass die allgemeine Schmerzwahrnehmung bei Patienten, die mittels Elektromagnetfeld eine zusätzliche Therapie erhielten, mit weniger Schmerzempfindung eine bessere Beweglichkeit aufwiesen (Tab. 3).

In der Placebogruppe (inaktives Elektromagnetfeld) hingegen wurde keine signifikante Verbesserung des durch Schmerzen beeinträchtigten Schlafes festgestellt. Dies ist insofern von besonderer Bedeutung als sich die Effizienz der elektromagnetischen Behandlung gegenüber den zusätzlich eingesetzten, an und für sich selbst schon sehr effizienten standardisierten Reha-Massnahmen bewähren musste. In Verbindung mit der überaus einfachen technischen Bedienung erscheinen derartige Feldapplikationen geradezu prädestiniert auch für die der klinischen Behandlung nachfolgenden häuslichen Rehabilitations- und Vorsorgemassnahmen.

Das Adhäsionsmolekül PECAM (Platelet-endothelial Cell Adhesion Mole-

Tab. 5. Auflistung signifikanter Korrelationen nach Spearman vor und nach 3-wöchiger Rehabilitation mit und ohne elektromagnetische Intervention

\begin{tabular}{|c|c|c|c|c|c|c|}
\hline \multicolumn{4}{|c|}{$\begin{array}{l}\text { Elektro-Magnetfeld aktiv + Rehabilitation } \\
\text { (Verumgruppe; } n=22 \text { ) }\end{array}$} & \multicolumn{3}{|c|}{$\begin{array}{l}\text { Elektro-Magnetfeld inaktiv + Rehabilitation } \\
\text { (Placebogruppe; } n=22 \text { ) }\end{array}$} \\
\hline \multicolumn{2}{|c|}{ Korrelation } & \multirow{2}{*}{$\begin{array}{c}\begin{array}{c}\text { Korrelations- } \\
\text { koeffizient }\end{array} \\
\text { rs } \\
\end{array}$} & \multirow{2}{*}{$\begin{array}{c}\text { Signifikanz } \\
\mathrm{p}\end{array}$} & \multicolumn{2}{|c|}{ Korrelation } & \multirow{2}{*}{$\begin{array}{c}\begin{array}{c}\text { Korrelations- } \\
\text { koeffizient }\end{array} \\
\text { rs } \\
\end{array}$} \\
\hline von & $\mathrm{zu}$ & & & von & $\mathrm{zu}$ & \\
\hline Ruheschmerz VAS & Druckschmerz & 0.48 & $<0,05$ & $\begin{array}{l}\text { Finger-Fußboden- } \\
\text { Abstand }\end{array}$ & Druckschmerz & 0.45 \\
\hline Ruheschmerz VAS & PECAM & 0.50 & $<0,05$ & Druckschmerz & $\begin{array}{l}\text { Roland \& Morris- } \\
\quad \text { Score }\end{array}$ & 0.59 \\
\hline Ruheschmerz VAS & $\begin{array}{l}\text { Roland \& Morris- } \\
\quad \text { Score }\end{array}$ & 0.42 & $<0,05$ & Ruheschmerz VAS & $\begin{array}{l}\text { Roland \& Morris- } \\
\text { Score }\end{array}$ & 0.73 \\
\hline $\begin{array}{l}\text { Lateraler Finger- } \\
\text { Fußboden-Abstand }\end{array}$ & Schonhaltung & 0.47 & $<0,05$ & & & \\
\hline Depression HADS & PSQI & 0.42 & $<0,05$ & & & \\
\hline Bewegungsschmerz VAS & PSQI & 0.41 & $<0,05$ & & & \\
\hline
\end{tabular}


cule 1) spielt als Mediator und Regulator von Zelladhäsionen und Zellmigrationen eine grosse Rolle sowohl bei den Interaktionen zwischen Endothelzellen untereinander und zwischen Endothelzellen und Leukozyten [32]. Aus tierexperimentellen Studien ist bekannt, dass ein rascher Einsatz von Antikörpern gegen PECAM-1 bei Infarktpatienten die Grösse des Infarktareals verringern kann, und weiter, dass bei Patienten mit akutem Myokardinfarkt eine erhöhte PECAM-Expression nachweisbar ist, die nach einer Thrombolyse signifikant abfällt [26].

Interessanterweise zeigte sich in unseren Untersuchungen eine signifikante Verminderung der auf geringeres thrombotisches Risiko hinweisenden Menge des Adhäsionsmoleküls PECAM in beiden Gruppen; eine Tatsache, die generell auf günstige Effekte im Gefässsystem während eines stationären Rehabilitationsaufenthaltes auch bei Patienten mit Erkrankungen des Stützund Bewegungsapparates hindeutet (Abb. 1). Auch das Adhäsionsmolekül P-Selektin (= Granule Membrane Protein-140/GMP-140) wird von aktivierten Endothelzellen, aber auch Thrombozyten exprimiert und als lösliches PSelektin in das Plasma abgegeben [25], in dem der enzymimmunologische Nachweis möglich ist. P-Selektin steht in Zusammenhang mit Plättchenaggregation und Atherosklerose [3]. Eine Verringerung von P-Selektin wäre in Zusammenhang mit einer Reduktion «frühatherosklerotischer» Prozessse am Endothel und vermindertem Risiko für kardiovaskuläre Ereignisse zu sehen. Obwohl relativ hohe Ausgangs-PSelektin-Konzentrationen im Mittelund im Medianwert im Verlauf von 3 Wochen zurückgingen (Abb. 1), verhindern sehr hohe individuelle Streuungen bei den kleinen Fallzahlen die Signifikanz und die Antwort, ob primär die Anwendung gepulster elektromagnetischer Felder oder der Rehabilitationsaufenthalt per se eine günstigen Effekt auf die P-Selektin-Expression ausübt. Es muss nochmals darauf hingewiesen werden, dass gerade Schmerzen ein hohes kardiovaskuläres Risiko darstellen und damit die protektive Wirkung der in dieser Studie signifikant verminderten biochemischen
Werte eine relevante Bedeutung für die Verbesserung im Laufe einer Rehabilitation aufweisen.

In Übereinstimmung mit einem Bericht der WHO zur Wirkung schwacher elektromagnetischer Felder [33] ergab sich im Rahmen unserer Studie kein einziger Hinweis auf Nebenwirkungen. Insbesondere sei darauf hingewiesen, dass in der Verumgruppe sogar eine Verbesserung der Schafqualität vorliegt. Wegen der vielerorts oft - möglicherweise aus Gründen einer nicht erkannten Bedeutung der Abhängigkeit biologischer Reaktionen von Qualität und Quantität des verantwortlichen physikalischen Reizes - unrichtig und oft abwertend geübten Pauschalisierung elektromagnetischer Feldwirkungen sei hier eigens betont, dass sich die hier vorgelegten Befunde ohne erneute und detaillierte Prüfung nicht verallgemeinernd auf andere (Elektro-)Magnetfeldapplikationen übertragen lassen [14].

\section{Fazit für die Praxis}

Nachdem diese spezielle nicht-invasive elektromagnetische Intervention (auf Grund der vorliegenden Ergebnisse) zusätzlich zu einem etablierten und effektiven Standardrehabilitationsprogramm nebenwirkungsfrei schwache positive additive Effekte zur Reduktion von Schmerzen, insbesondere auf den durch chronischen Schmerz beeinträchtigten Schlaf, bewirken dürfte, kann man bei chronischem Kreuzschmerz teilweise günstige Effekte erwarten. Dieser Effekt ist zwar gering, aber möglicherweise besser im Langzeiteffekt. Angesichts derzeitiger Forschungs- und Entwicklungsarbeiten zur Optimierung und Effizienz der dieser Behandlung zugrundeliegenden Signalformen erscheinen Verbesserungen in der Wirksamkeit, auch hinsichtlich der Breite ihrer Einsatzmöglichkeiten in der medizinischen und klinischen Praxis, im Rehabilitationswesen, im Sport, aber auch in der Heimanwendung möglich.

\section{Danksagung}

Wir danken den beteiligten Institutionen, alIen Mitarbeitern und Patienten für die Mitwirkung und Unterstützung bei der Durchführung dieser Studie.

\section{Literatur}

1. Aaron RK, Boyan BD, Ciombor DM, Schwartz Z, Simon BJ: Stimulation of growth factor synthesis by electric and electromagnetic fields. Clin Orthop Relat Res 2004;419:30-7.

2. Al-Awami M, Willfort A, Gschwandtner ME, Minar E: Treatment of raynaud's phenomenon with pulsed electromagnetic fields. International Angiology - The Journal of Vascular Biology, Medicine and Surgery 2004;23(Suppl. 1):193.

3. Andre P: P-selectin in atherothrombosis: new directions for monitoring the pro-inflammatory activity of platelets. Clinical Laboratory International 2005;29(3):14-16.

4. Bullinger M, Kirchberger I: SF-36 Fragebogen zum Gesundheitszustand - Handanweisung. Hogrefe Verlag, Göttingen 1998

5. Buysse DJ, Reynolds CF, Monk Th, Berman SR, Kupfer DJ: The Pittsburgh Sleep Quality Index: A new instrument for psychiatric practice and research. Psychiatry Res 1988;28: 193-213.

6. Carpenter DO and Aryapetyan S: Biological Effects of Electric and Magnetic Fields: Sources and mechanism (Vol 1): Beneficial and Harmful Effects (Vol 2). Academic Press 1994, ISBN 012160262.

7. Ciombor D, Lester G, Aaron R, Neame P, Caterson B: Low frequency EMF regulates chondrocyte differentiation and expression of matrix proteins. J Orthop Res 2002;20:40-50.

8. Herrmann C, Scholz KH, Kreuzer H : Psychologic screening of patients of a cardiologic acute care clinic with the German version of the Hospital Anxiety and Depression Scale. Psychother Psychosom Med Psychol 1991; 41(2):83-92

9. Herrmann C: International experiences with the Hospital Anxiety and Depression Scale a review of validation data and clinical results. J Psychosom Res 1997;42(1):17-41.

10. Gschwandtner ME, Al-Awami M, Haumer M, Maric S, Mlekusch W, Willfort A, Minar E: Effect of electromagnetic fields (Bemer 3000 ) on microcirculation in ulcers and adjacent skin. International Angiology - The Journal of Vascular Biology, Medicine and Surgery 2004; 23 (Suppl. 1):70

11. Jelínek R, Bláha J and Dbaly J (2009): Electromagnetic signals modify response to teratogens. 3rd Int. Emphyspace Symposium on Bioelectromagnetic Energy Regulation, 2002, Bad-Windsheim, Germany.

12. Kafka WA (1998): Vorrichtung und elektrisches oder elektromagnetisches Signal zur Beeinflussung biologischer Systeme. Europäische Patentanmeldung 98119944.1 v. 21.10.1998.

13. Kafka WA: Bio-Elektro-Magnetische-EnergieRegulation: Das physikalische Konzept und sein Einsatz bei Schmerz auslösenden Störungen. In: Bernatzky G. (ed.): Komplementäre Methoden in der Schmerzbehandlung. Springer Verlag Berlin-Heidelberg-New York 2007, S. 300-321.

14. Kafka WA: Elektro-Magnetfeldtherapie. In: Likar R, Bernatzky G, Märkert D, Ilias W (ed.): Schmerztherapie in der Pflege: Schulmedizinische und komplementäre Methoden. Springer Verlag Berlin-Heidelberg-NewYork 2009 (in press). 
15. Kafka WA und Preissinger $M$ : Verbesserte Wundheilung durch gekoppelte, speziell gepulste Elektromagnetfeld- und LED-LichtTherapie am Beispiel vergleichender Untersuchungen an standardisierten Wunden nach Ovarioektomie bei Katzen (felidae). In: Edwin Ganster (ed.): Österreichische Gesellschaft der Tierärzte (ÖGT), Kleintiertage-Dermatologie 02.-03. März 2002, Salzburg Congress.

16. Kafka WA, Schütze N, Walther M: Einsatz extrem niederfrequent (BEMER-typisch) gepulster schwacher elektromagnetischer Felder im Bereich der Orthopädie (Application of extreme low frequent (BEMER type) pulsed electromagnetic fields in orthopedics) Orthopädische Praxis 2005;41(1):22-24.

17. Kafka WA, Spodaryk K: Effects of extremely weak BEMER 3000 type pulsed electromagnetic fields on red blood metabolism and hemoglobin oxygen affinity. Fizoterapia 2003; 11(3):24-31. ISSN 1230-8323.

18. Klopp R, Niemer W: Einfluss eines pulsierenden elektromagnetischen Feldes mit vasomotorischer Stimulation auf einen eingeschränkten Funktionszustand. Mikrozirkulation Komplement. integr. Med. 08/2007.

19. Lohaus A, Schmitt GM: KKG Fragebogen zur Erhebung von Kontrollüberzeugungen zu Krankheit und Gesundheit. Hogrefe Verlag, Göttingen 1989.

20. Michels-Wakili S, Kafka WA: Bemer 3000 Type Pulsed Low-Energy Electromagnetic Fields Reduce Dental Anxiety: A Randomized Placebo-Controlled Single-Blind Study. 10th International Dental Congress on Modern Pain Control IFDAS, June 2003, Edinburgh, Scotland.

21. Quittan M, Schuhfried $O$, Wiesinger GF, Fialka-Moser V: Klinische Wirksamkeiten der Magnetfeldtherapie - eine Literaturübersicht. Acta Medica Austriaca 2000:3:61-68.

22. Rihova B, Dbaly J, Jelinek R, Kafka WA: Exposure to special pulsed, weak electromagnetic fields does not accelerate the growth of mouse EL4 T cell lymphoma. 2007 (in prep).

23. Roland M, Morris R: A study of the natural history of back pain. Part I: development of a reliable and sensitive measure of disability in low back pain. Spine 1983;8:141-144.

24. Ruoff G: Effekte elektromagnetischer Felder auf biologische Systeme und Expressionsmuster von Wachstumsfaktoren. Ein Review. Schweiz Zschr GanzheitsMedizin 2008;20(6): 347-353.

25. Schuhfried O, Vacariu G, Rochowanski H, Serek M, Fialka-Moser V: The effects of lowdosed and high-dosed low-frequency electromagnetic fields on microcirculation and skin temperature in healthy subjects. International journal of sports medicine 2005;26(10): 886-90.

26. Serebruany VL, Gurbel PA: Effect of Thrombolytic Therapy on Platelet Expression and Plasma Concentration of PECAM-1 (CD31) in Patients with Acute Myocardial Infarction. Arteriosclerosis, Thrombosis, and Vascular Biology 1999;19:153-158.

27. Siegmeth W: Epidemiologie und Risikofaktoren des chronischen Rückenschmerzes. In: Wagner E, Ulreich A. (Hrsg): Rehabilitation beim chronisch unspezifischen Kreuzschmerz. Man Crossmedia Verlag, Wien 2003.

28. Spodaryk K: The effect of extremely weak electromagnetic field treatments upon signs and symptoms of delayed onset of muscle soreness: A placebo controlled clinical double blind study. Medicina Sportiva 2002;6:19-25.

29. Spodaryk K and Kafka WA: The influence of extremely weak pulsed electromagnetic field on ratings of perceived exertion (RPE) at ven- tilatory threshold. International Journal of Rehabilitation Research (EFRR), 2004;27(1): 144. ISSN 0342-5282.

30. Vallbona C, Hazlewood CF, Jurida G: Response of pain to static magnetic fields in postpolio patients: A double-blind pilot study. Arch Phys Med Rehabil 1983;78:1200-1203.

31. Walther M, Mayer F, Kafka WA, Schütze N: Effects of weak, low frequency pulsed electromagnetic fields (BEMER type) on gene expression of human mesenchymal stem cells and chondrocytes: an in vivo study. Electromagnetic Biology and Medicine 2007; 26:179-190. Manuscript ID: 257936.

32. Wang $Y$, Sheibani N: PECAM-1 isoform-specific activation of MAPK/ERKs and small GTPases: Implications in inflammation and angiogenesis. J Cell Biochem 2006;26 [Epub ahead of print].

33. Gesundheitliche Wirkungen im Überblick: Was passiert, wenn Menschen elektromagnetischen Feldern ausgesetzt werden? http://www.who.int/peh-emf/about/Whatis EMF/en/index1.htm

34. Zigmond AS, Snaith RP: The hospital anxiety and depression scale. Acta Psychiatr Scand 1983;67(6):361-370.

\section{Disclosure Statement}

Die Studie wurde finanziell unterstützt durch die Firma Innomed International AG, FL-Triesen. Die Präsentation des Themas ist unabhängig und die Darstellung der Inhalte produktneutral.

\section{Korrespondenzadresse}

Univ.-Prof. Dr. Günther Bernatzky

Naturwissenschaftliche Fakultät

Fachbereich für Organismische Biologie

Arbeitsgruppe für "Neurodynamics \&

Neurosignaling", Universität Salzburg

Hellbrunnerstrasse 34, A-5020 Salzburg

guenther.bernatzky@sbg.ac.at 